\title{
Cancer-related inflammation
}

\author{
Alberto Mantovani ${ }^{1,2}$, Paola Allavena ${ }^{1}$, Antonio Sica ${ }^{3}$ \& Frances Balkwill ${ }^{4}$
}

The mediators and cellular effectors of inflammation are important constituents of the local environment of tumours. In some types of cancer, inflammatory conditions are present before a malignant change occurs. Conversely, in other types of cancer, an oncogenic change induces an inflammatory microenvironment that promotes the development of tumours. Regardless of its origin, 'smouldering' inflammation in the tumour microenvironment has many tumour-promoting effects. It aids in the proliferation and survival of malignant cells, promotes angiogenesis and metastasis, subverts adaptive immune responses, and alters responses to hormones and chemotherapeutic agents. The molecular pathways of this cancer-related inflammation are now being unravelled, resulting in the identification of new target molecules that could lead to improved diagnosis and treatment.

Links between cancer and inflammation were first made in the nineteenth century, on the basis of observations that tumours often arose at sites of chronic inflammation and that inflammatory cells were present in biopsied samples from tumours ${ }^{1}$. The idea that these processes are connected was out of favour for more than a century, but there has been a recent resurgence in interest. Several lines of evidence ${ }^{1-4}$ (Box 1) based on a range of findings, from epidemiological studies of patients to molecular studies of genetically modified mice - have led to a general acceptance that inflammation and cancer are linked.

Epidemiological studies have shown that chronic inflammation predisposes individuals to various types of cancer. It is estimated that underlying infections and inflammatory responses are linked to $15-20 \%$ of all deaths from cancer worldwide ${ }^{1}$. There are many triggers of chronic inflammation that increase the risk of developing cancer. Such triggers include microbial infections (for example, infection with Helicobacter pylori is associated with gastric cancer and gastric mucosal lymphoma), autoimmune diseases (for example, inflammatory bowel disease is associated with colon cancer) and inflammatory conditions of unknown origin (for example,

\section{Box 1 The evidence that links cancer and inflammation}

- Inflammatory diseases increase the risk of developing many types of cancer (including bladder, cervical, gastric, intestinal, oesophageal, ovarian, prostate and thyroid cancer).

- Non-steroidal anti-inflammatory drugs reduce the risk of developing certain cancers (such as colon and breast cancer) and reduce the mortality caused by these cancers.

- Signalling pathways involved in inflammation operate downstream of oncogenic mutations (such as mutations in the genes encoding RAS, MYC and RET).

- Inflammatory cells, chemokines and cytokines are present in the microenvironment of all tumours in experimental animal models and humans from the earliest stages of development.

- The targeting of inflammatory mediators (chemokines and cytokines, such as TNF- $\alpha$ and IL-1 $\beta$ ), key transcription factors involved in inflammation (such as NF-kB and STAT3) or inflammatory cells decreases the incidence and spread of cancer.

- Adoptive transfer of inflammatory cells or overexpression of inflammatory cytokines promotes the development of tumours. prostatitis is associated with prostate cancer). Accordingly, treatment with non-steroidal anti-inflammatory agents decreases the incidence of, and the mortality that results from, several tumour types ${ }^{5-7}$.

The hallmarks of cancer-related inflammation include the presence of inflammatory cells and inflammatory mediators (for example, chemokines, cytokines and prostaglandins) in tumour tissues, tissue remodelling and angiogenesis similar to that seen in chronic inflammatory responses, and tissue repair. These signs of 'smouldering' inflammation ${ }^{2}$ are also present in tumours for which a firm causal relationship to inflammation has not been established (for example, breast tumours). Indeed, inflammatory cells and mediators are present in the microenvironment of most, if not all, tumours, irrespective of the trigger for development.

Studies of genetically modified mice, adoptive-transfer experiments in mice, and analyses of human tumours have allowed researchers to begin to unravel the molecular pathways that link inflammation and cancer. Here we review current knowledge of the molecular and cellular pathways that link inflammation and cancer, and we describe how these pathways suppress effective antitumour immunity during tumour progression. We also discuss how cancer-related inflammation affects many aspects of malignancy, including the proliferation and survival of malignant cells, angiogenesis (which is required for the survival of cells within tumours of a certain size), tumour metastasis, and tumour response to chemotherapeutic drugs and hormones.

Advances in understanding the genetic pathways involved in cancer have led to the development of a range of therapies that target malignant cells. Understanding the pathways involved in cancer-related inflammation could enable the development of synergistic therapies that target 'the other half of the tumour' - that is, the inflammatory components of the microenvironment. Preclinical and early clinical studies are now suggesting how this might be achieved. However, despite considerable progress, important questions remain unanswered, as we discuss in the final section of this Review.

\section{Connecting inflammation and oncogenes}

The connection between inflammation and cancer can be viewed as consisting of two pathways: an extrinsic pathway, driven by inflammatory conditions that increase cancer risk (such as inflammatory bowel disease); and an intrinsic pathway, driven by genetic alterations that

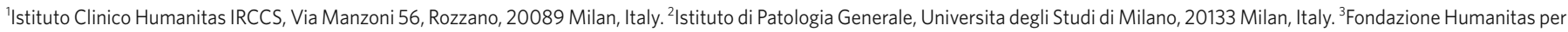

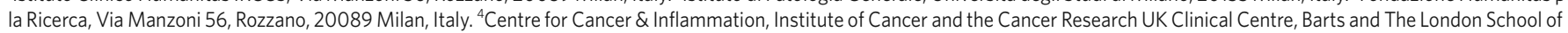
Medicine and Dentistry, London EC1M 6BQ, UK. 
cause inflammation and neoplasia (such as oncogenes) (Fig. 1). The intrinsic pathway was uncovered when addressing why inflammatory cells and mediators are present in the microenvironment of most, if not all, tumours and therefore are present in cases for which there is no epidemiological basis for inflammation. This finding raised the question of whether the genetic events that cause neoplasia in these cases are responsible for generating an inflammatory environment. This question has been addressed only recently, by using preclinical and clinical settings in which various oncogenetic mechanisms can be assessed.

An example of this type of study involves protein tyrosine kinases, which are encoded by prototypical transforming oncogenes. A useful clinical setting in which to explore the connection between these oncogenes and an inflammatory microenvironment is human papillary thyroid carcinoma. Rearrangement of the chromosome on which the gene encoding the protein tyrosine kinase RET is located (also referred to as the RET/PTC rearrangement) is a frequent early event in the pathogenesis of papillary thyroid carcinoma and is a necessary and sufficient event for this cancer to develop. In an appropriate cellular context, which is provided by freshly isolated human thyrocytes, the activation of RET induces a transcriptional program that is similar to that which occurs during inflammation ${ }^{8}$ (Fig. 2). The transcriptome of cells in which RET has been activated includes messenger RNA encoding various factors: colony-stimulating factors (CSFs), which promote the survival of leukocytes and their recruitment from the blood to the tissues; interleukin $1 \beta$ (IL-1 $\beta$ ), one of the main inflammatory cytokines; cyclooxygenase 2 (COX2), which is frequently expressed by cancerous cells and is involved in the synthesis of prostaglandins; chemokines that can attract monocytes and dendritic cells (CC-chemokine ligand 2 (CCL2) and CCL20); chemokines that promote angiogenesis (such as IL-8; also known as CXC-chemokine ligand 8 (CXCL8)); the chemokine receptor CXC-chemokine receptor 4 (CXCR4), which binds to CXCL12; extracellular-matrix-degrading enzymes; and the adhesion molecule lymphocyte selectin (L-selectin). Key protein components of the RET-activated 'inflammatory' program were found in tumour specimens taken by biospy, and larger amounts of these inflammatory molecules were found in the primary tumours of patients with lymph-node metastasis than in primary tumours in the absence of lymph-node metastasis ${ }^{8,9}$. These results show that an early genetic event that is necessary and sufficient for the development of a human tumour directly promotes the build-up of an inflammatory microenvironment ${ }^{8}$. Although these $\mathrm{e}^{8,9}$ and other results ${ }^{10}$ connect the activation of protein-tyrosine-kinase-encoding oncogenes to inflammation, the precise roles of the various components of the inflammatory microenvironment in the progression of tumours remain to be defined.

Members of the RAS family are the most frequently mutated dominant oncogenes in human cancer, and activated oncogenic components of the RAS-RAF signalling pathway, in turn, induce the production of tumour-promoting inflammatory chemokines and cytokines ${ }^{11-13}$. Another oncogene, $M Y C$, encodes a transcription factor that is overexpressed in many human tumours; deregulated expression of this gene initiates and maintains key aspects of the tumour phenotype. In addition to promoting cell-autonomous proliferation, MYC instructs the remodelling of the extracellular microenvironment, with inflammatory cells and mediators having important roles in this process. In a mouse model of MYC-dependent cancer of the $\beta$-cells (which are insulin-producing pancreatic islet cells), the first wave of angiogenesis results from MYC-induced production of the inflammatory cytokine IL- $1 \beta^{14}$. The MYC-activated transcriptional program also elicits the production of several chemokines that recruit mast cells. Mast cells have long been known to drive angiogenesis, and in this case (following IL-1 $\beta$ ) they sustain new blood-vessel formation and tumour growth ${ }^{15}$.

These studies of RAS family members and MYC show that dominant oncogenes promote the formation of a tumour-promoting tissue microenvironment (the intrinsic pathway), but the findings do not address the issue of the interplay between oncogenes and inflammatory conditions that increase the risk of developing cancer (the extrinsic pathway) (Fig. 1). This interplay is likely to occur in pancreatic carcinoma, in which both pancreatitis and mutations in the gene encoding K-RAS
Extrinsic pathway

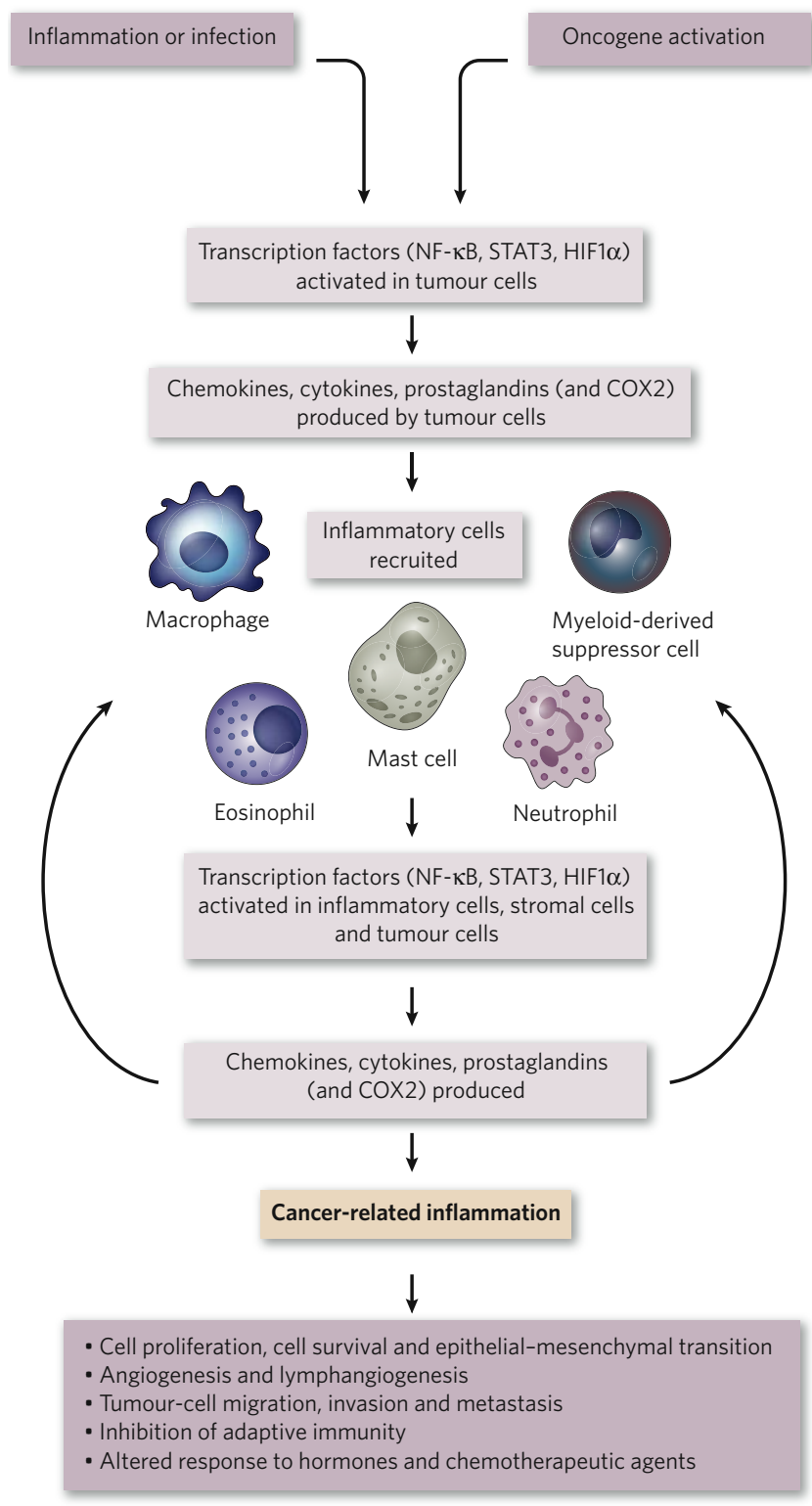

Figure 1 | Pathways that connect inflammation and cancer. Cancer and inflammation are connected by two pathways: the intrinsic pathway and the extrinsic pathway. The intrinsic pathway is activated by genetic events that cause neoplasia. These events include the activation of various types of oncogene by mutation, chromosomal rearrangement or amplification, and the inactivation of tumour-suppressor genes. Cells that are transformed in this manner produce inflammatory mediators, thereby generating an inflammatory microenvironment in tumours for which there is no underlying inflammatory condition (for example, breast tumours). By contrast, in the extrinsic pathway, inflammatory or infectious conditions augment the risk of developing cancer at certain anatomical sites (for example, the colon, prostate and pancreas). The two pathways converge, resulting in the activation of transcription factors, mainly nuclear factor- $\kappa \mathrm{B}(\mathrm{NF}-\kappa \mathrm{B})$, signal transducer and activator of transcription 3 (STAT3) and hypoxia-inducible factor $1 \alpha$ (HIF1 $\alpha)$, in tumour cells. These transcription factors coordinate the production of inflammatory mediators, including cytokines and chemokines, as well as the production of cyclooxygenase 2 (COX2) (which, in turn, results in the production of prostaglandins). These factors recruit and activate various leukocytes, most notably cells of the myelomonocytic lineage. The cytokines activate the same key transcription factors in inflammatory cells, stromal cells and tumour cells, resulting in more inflammatory mediators being produced and a cancer-related inflammatory microenvironment being generated. Smouldering cancer-related inflammation has many tumour-promoting effects. 


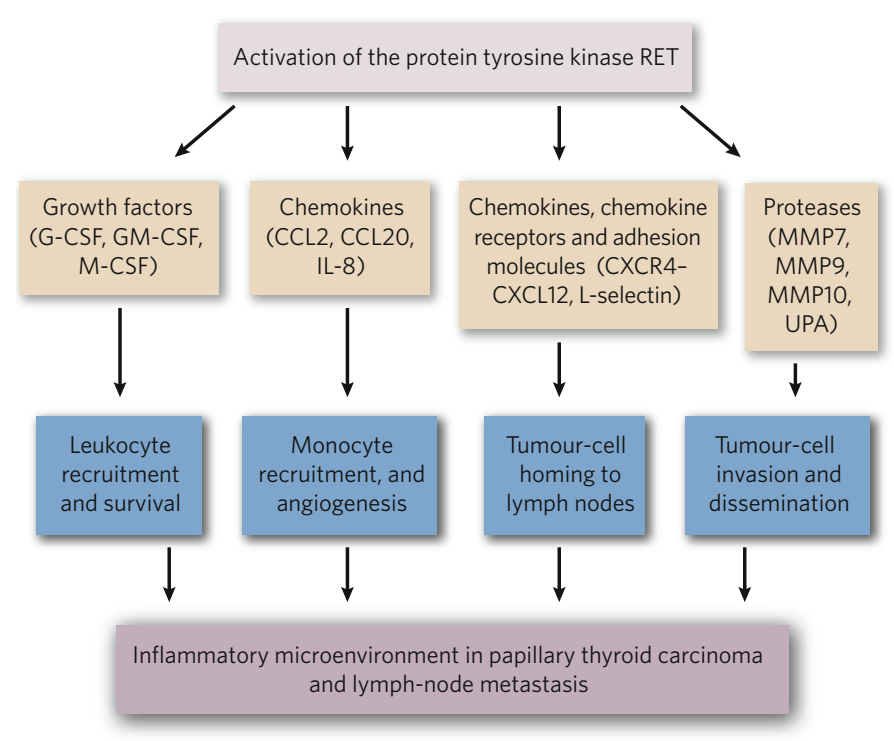

Figure 2 Oncogenes and cancer-related inflammation. A class of oncogenes encodes protein tyrosine kinases that are persistently activated in a ligand-independent manner as a result of mutation or chromosomal rearrangement. RET is representative of these activated oncogenes. A chromosomal rearrangement that affects $R E T$ is a frequent early event in the pathogenesis of human papillary thyroid carcinoma. In human thyrocytes maintained in short-term culture, RET activates an inflammatory transcriptional program, the components of which are found in tumours obtained from patients. The inflammatory mediators that are produced in response to this program, together with their effects on tumour cells and inflammatory cells, are indicated. CCL, CC-chemokine ligand; CSF, colony-stimulating factor; CXCL12, CXC-chemokine ligand 12; CXCR4, CXC-chemokine receptor 4; IL-8, interleukin 8; L-selectin, lymphocyte selectin; MMP, matrix metalloproteinase; UPA, urokinase-type plasminogen activator.

are frequently found. In a relevant mouse model, adult mice are resistant to mutated Kras-induced pancreatic carcinogenesis ${ }^{11}$. Both mild chronic pancreatitis (possibly mirroring the clinical epidemiology) and mutated Kras are required to induce pancreatic intra-epithelial neoplasia and invasive ductal carcinoma ${ }^{11}$. Thus, although the RAS-RAF pathway ${ }^{12,13}$ can drive tumour-promoting inflammation to a certain extent, an extrinsic inflammatory condition (pancreatitis) is needed to drive carcinogenesis in mice and presumably in humans.

Tumour-suppressor proteins can also regulate the production of inflammatory mediators. Examples of such proteins are von Hippel-Lindau tumour suppressor (VHL), transforming growth factor- $\beta$ (TGF- $\beta$ ) and phosphatase and tensin homologue $(\mathrm{PTEN})^{16-20}$. VHL is a component of a molecular complex that targets the transcription factor hypoxia-inducible factor $1 \alpha(\mathrm{HIF} 1 \alpha)$ for degradation. HIF1 $\alpha$ promotes the cellular and tissue response to hypoxia, including angiogenesis. It also functionally interacts with the transcription factor nuclear factor- $\kappa \mathrm{B}(\mathrm{NF}-\kappa \mathrm{B})$ (discussed later), resulting in the production of the major inflammatory cytokine tumournecrosis factor- $\alpha$ (TNF- $\alpha$ ) and the chemokine receptor CXCR4 in human renal-cell carcinoma cells, as well as in other malignant cell types ${ }^{2,16,19,20}$. The production of CXCR4 is particularly relevant, because CXCR4 expression is frequently upregulated in human cancer and CXCR4 is involved in metastasis ${ }^{16}$ (discussed later).

Recent evidence from a mouse model of cancer links TGF- $\beta$, a tumoursuppressor protein that is frequently involved in the progression of human cancer, to tumour-promoting inflammation ${ }^{21}$. In an animal model of breast carcinoma, inactivation of the gene encoding the type II TGF- $\beta$ receptor (which initiates carcinogenesis by preventing the actions of TGF- $\beta$ ) unleashes the production of CXCL5 and CXCL12. These chemokines attract cells known as myeloid-derived suppressor cells (MDSCs), which belong to the myelomonocytic lineage. MDSCs are potent suppressors of the adaptive immune response to tumours and directly facilitate metastasis. It will be important to assess whether this pathway occurs in human tumours in which the TGF- $\beta$ receptor is involved.

Thus, the various types of oncogene (such as those encoding protein tyrosine kinases, RAS and RAF, transcription factors and tumoursuppressor proteins), irrespective of their molecular class or mode of action, all coordinate inflammatory transcriptional programs. And these oncogene-coordinated inflammatory responses seem to have aspects in common: a link to angiogenesis, and the recruitment of cells of myelomonocytic origin. Several issues remain to be fully elucidated, including which components of inflammation are essential and which are redundant, the relative importance of these components in carcinogenesis in different tissues, and the relevance of these components to different types of cancer in humans.

\section{Key factors in cancer-related inflammation}

In the panoply of molecules involved in cancer-related inflammation, key endogenous (intrinsic) factors can be identified. These include transcription factors (such as NF- $\mathrm{KB}$ and signal transducer and activator of transcription 3 (STAT3)) and major inflammatory cytokines (such as IL-1 $\beta$, IL- 6, IL-23 and TNF- $\alpha)^{4,22-26}$. The main inflammatory cytokines were discussed earlier, so this section focuses on transcription factors.

$\mathrm{NF}-\kappa \mathrm{B}$ is a key coordinator of innate immunity and inflammation, and has emerged as an important endogenous tumour promoter ${ }^{4}$. NF- $\kappa \mathrm{B}$ is crucial both in the context of tumour or potential tumour cells and in the context of inflammatory cells. In these cell types, NF- $\kappa \mathrm{B}$ operates downstream of the sensing of microorganisms or tissue damage by the Toll-like receptor (TLR)-MyD88 signalling pathway, and by signalling pathways mediated by the inflammatory cytokines TNF- $\alpha$ and IL-1 $\beta$. In addition, NF- $\kappa \mathrm{B}$ can be activated as a result of cell-autonomous genetic alterations (amplification, mutations or deletions) ${ }^{27}$ in tumour cells.

In tumour cells and epithelial cells at risk of transformation by carcinogens, as well as in inflammatory cells, NF- $\mathrm{kB}$ activates the expression of genes encoding inflammatory cytokines, adhesion molecules, enzymes in the prostaglandin-synthesis pathway (such as COX2), inducible nitric oxide synthase (iNOS; also known as NOS2) and angiogenic factors. In addition, one of the important functions of NF- $\kappa \mathrm{B}$ in tumour cells or cells targeted by carcinogenic agents is promoting cell survival, by inducing the expression of anti-apoptotic genes (such as BCL2). There is also accumulating evidence of interconnections and compensatory pathways between the NF- $\kappa \mathrm{B}$ and HIF $1 \alpha$ systems ${ }^{28-30}$, linking innate immunity to the response to hypoxia.

There is unequivocal evidence that NF- $\kappa \mathrm{B}$ is involved in tumour initiation and progression in tissues in which cancer-related inflammation typically occurs (such as the gastrointestinal tract and the liver) ${ }^{31,32}$. This evidence is based on various genetic studies, such as tissue-specific targeting of genes that encode components of the IкB kinase (IKK) complex. This complex phosphorylates inhibitor of NF- $\kappa \mathrm{B}$ (I $\kappa \mathrm{B})$, causing it to dissociate from NF- $\mathrm{BB}$ and allowing NF- $\kappa \mathrm{B}$ to translocate to the nucleus, where it can exert its function as a transcription factor. It should be noted that genetic targeting of NF- $\kappa \mathrm{B}$ in liver epithelial cells can have divergent effects in different models of carcinogenesis, possibly depending on the balance between promoting apoptosis that has already been initiated and triggering compensatory cell proliferation ${ }^{32,33}$.

The NF- $\kappa B$ pathway is tightly controlled by inhibitors that function at various stages of the pathway. An example is TIR8 (also known as SIGIRR), a member of the IL-1-receptor family. TIR8 has a single immunoglobulin domain, a long cytoplasmic tail, and a Toll/IL-1 receptor (TIR) domain that differs from that of other members of the IL-1receptor family. It inhibits signalling through TLRs and the IL-1 receptor and is highly expressed in the intestinal mucosa. Deficiency in the gene that encodes TIR 8 is associated with increased susceptibility to intestinal inflammation and carcinogenesis ${ }^{34,35}$. Thus, the balance of inhibitors and activators tunes the extent to which the NF- $\kappa$ B pathway operates as an endogenous tumour promoter.

Support for the connection between cancer and inflammation is further strengthened by studies of the role of NF- $\kappa$ B in tumour-infiltrating leukocytes. For example, by using the strategy mentioned above, 
myeloid-lineage-specific inactivation of the gene encoding IKK- $\beta$ was found to inhibit cancer-related inflammation in the intestine, as well as colitis-associated cancer, unequivocally showing that inflammatory cells are involved in carcinogenesis in this tissue ${ }^{31}$. In established, advanced tumours, which typically have a microenvironment of smouldering inflammation ${ }^{2}$, tumour-associated macrophages (TAMs) have delayed and defective NF- $\kappa \mathrm{B}$ activation ${ }^{36}$. Evidence suggests that homodimers of the $\mathrm{p} 50$ subunit of NF- $\kappa \mathrm{B}$ (a negative regulator of the NF- $\kappa \mathrm{B}$ pathway) are responsible for this sluggish activation of NF- $\kappa B$ in TAMs and for the protumour phenotype of these cells ${ }^{37}$. Thus, NF- $\kappa$ B seems to function as a 'rheostat' whose function can be tuned to different levels, a property that enables the extent of inflammation to be regulated. Such regulation allows the vigorous inflammation (for example, in inflammatory bowel disease) that predisposes individuals towards developing cancer to be sustained, and enables TAMs to sustain the smouldering inflammatory microenvironment present in established metastatic neoplasia.

Similar to NF- $\kappa \mathrm{B}, \mathrm{STAT} 3$ is a point of convergence for numerous oncogenic signalling pathways ${ }^{22}$. This transcription factor is constitutively activated both in tumour cells and in immune cells, and is involved in oncogenesis and inhibition of apoptosis ${ }^{38}$. The activation of STAT3 in tumour cells has also been shown to increase the capacity of tumours to evade the immune system, by inhibiting the maturation of dendritic cells $\mathrm{s}^{39}$ and suppressing the immune response $\mathrm{e}^{40}$.

\section{Tumour-infiltrating leukocytes}

A leukocyte infiltrate, varying in size, composition and distribution, is present in most, if not all, tumours. Its components include TAMs and related cell types, mast cells and T cells. There is evidence (based on adoptive-transfer studies, cell-depletion studies, clinical correlations and gene-manipulation studies) that each of these bone-marrowderived components can be involved in carcinogenesis and/or tumour invasion and metastasis ${ }^{41-44}$.

In this section, we focus on TAMs. TAMs are an important component of the leukocyte infiltrate, and studies of TAMs formed the basis for the model that leukocyte infiltrates are involved in tumour progression. Plasticity and diversity are hallmarks of mononuclear phagocytes. In addition to conventional TAMs, related cell populations (for example, a TIE2-expressing monocyte subset, MDSCs and myeloid dendritic cells) have been linked to a protumour inflammatory microenvironment ${ }^{45,46}$. The ontogenetic relationship between these cell types and their relative importance in the context of tumours remain to be elucidated.

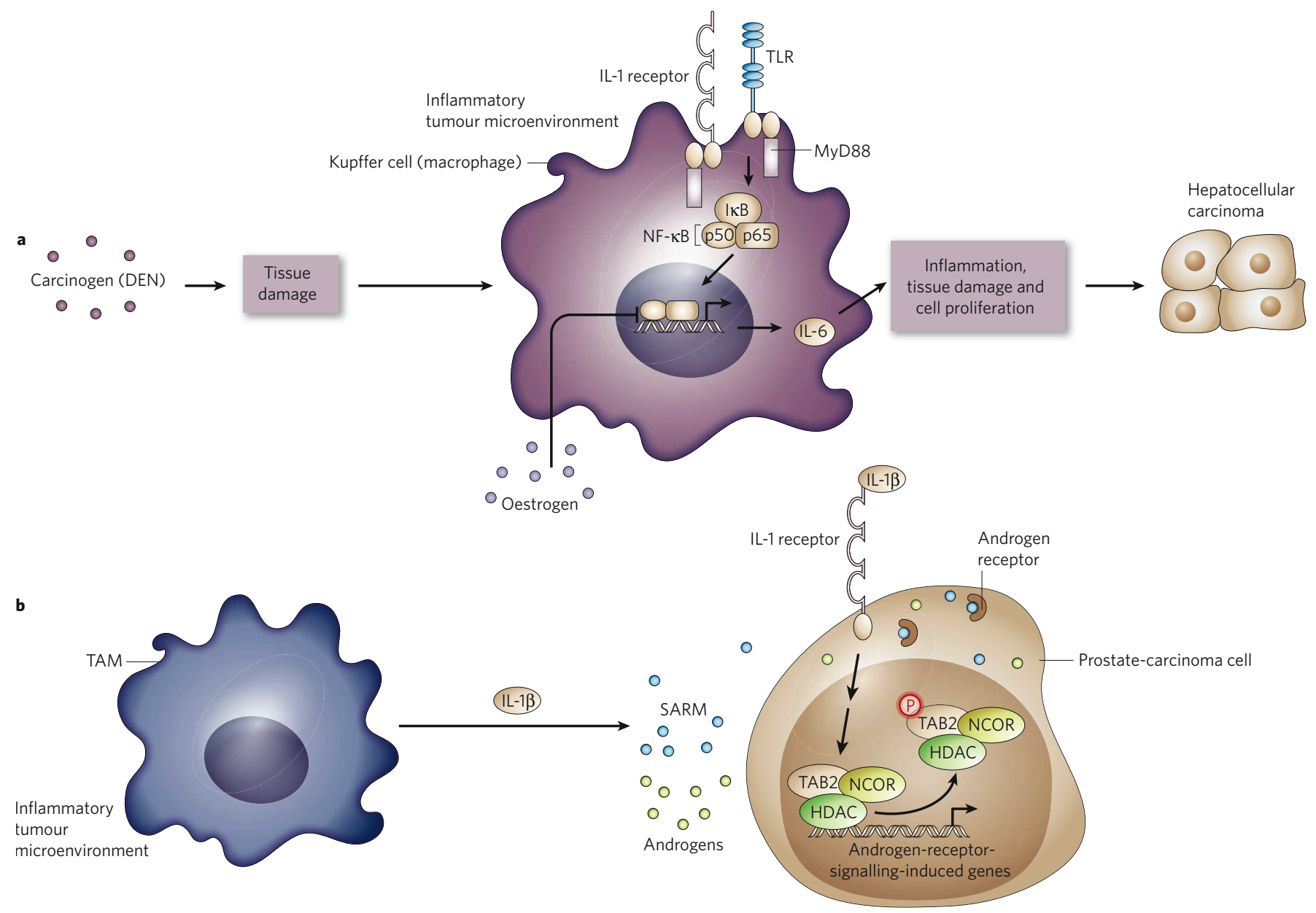

Figure 3 | Hormones and inflammation. Hormones and inflammation are each involved in a classic pathway that promotes tumour development. a, Liver. The carcinogen diethylnitrosamine (DEN) can activate the MyD88 signalling pathway in Kupffer cells (a type of macrophage) in the liver (presumably through TLRs or IL-1 receptors), resulting in the production of IL-6 (ref. 61). IL-6, in turn, promotes inflammation, tissue damage, compensatory cell proliferation and, ultimately, formation of a liver tumour. Oestrogens interfere with the activity of NF- $\mathrm{kB}$, the transcription factor that regulates IL-6 production, and thereby protect against carcinogenesis ${ }^{61}$. b, Prostate. Patients with prostate tumours are often treated with drugs known as selective androgen-receptor modulators (SARMs), which reduce the growth-promoting effects of male sex hormones (androgens) on the tumour. TAMs can, however, produce IL-1 $\beta$, which results in the desired action of SARMs being reversed, from inhibitors of androgen-receptorsignalling-induced gene expression (as intended) to activators. This process involves TAB2 (TGF- $\beta$-activated kinase 1 (TAK1)-binding protein 2 ), a sensor of inflammatory signals. TAB2 is a component of a co-repressor complex that includes the nuclear-receptor co-repressor (NCOR) and a histone deacetylase (HDAC). IL-1 $\beta$-mediated signalling results in the phosphorylation of TAB2, thereby lifting the repression of transcription. SARMs thus promote tumour-cell proliferation instead of inhibiting it. (Figure modified, with permission, from ref. 92.) 
TAMs and related cell types in mouse and human tumours generally have an M2 phenotype, which is oriented towards promoting tumour growth, remodelling tissues, promoting angiogenesis and suppressing adaptive immunity ${ }^{45,47}$. Signals that are derived from regulatory $\mathrm{T}$ cells present in tumours or from the tumour cells themselves (including macrophage CSF (M-CSF), IL-10 and TGF- $\beta)^{45,47,48}$ might account for this polarization to the $\mathrm{M} 2$ phenotype of macrophages that have been recruited into tumours. However, a stringent analysis of the molecular mechanisms responsible for this functional polarization during tumour progression has not been carried out.

Leukocyte infiltration is also interconnected with angiogenesis, which is required in tumours of a certain size. The pro-angiogenic protein vascular endothelial growth factor (VEGF) and related molecules are potent monocyte attractants and contribute to the recruitment of monocytes into primary tumours and the metastatic niche $e^{46,49-51}$. In turn, the recruited leukocytes provide an indirect (VEGF-independent) pathway of angiogenesis, through the secretion of pro-angiogenic factors ${ }^{41,51-55}$. It is possible therefore that the inhibition of leukocyte recruitment will improve the activity of current anti-angiogenic therapies for patients with cancer.

\section{Cancer-related inflammation and adaptive immunity}

There is strong evidence from genetic studies of mouse models that cells of the adaptive immune system carry out surveillance and can eliminate nascent tumours (a process called immuno-editing) ${ }^{56}$. Innate immune responses, which manifest as inflammation, are crucial for the initiation of adaptive immune responses. Therefore, the seemingly divergent effects of inflammation and immuno-editing are paradoxical. Yet, a recent study in mice shows that the TLR adaptor MyD88 (which is involved in innate immune responses) has a key role in promoting tumour development and that inflammation-induced carcinogenesis and immuno-editing can occur in the same tumour model ${ }^{57}$.

Another aspect of the complex interplay between adaptive immunity and cancer-related inflammation was shown by studies in a mouse model of cancer caused by human papilloma virus. In this system, antibodies are deposited in the tumour stroma. These antibodies then function as a 'remote-control system', binding to unidentified molecules in the extracellular matrix and thereby activating inflammatory responses that promote cancer progression ${ }^{58}$.

In clinically overt neoplasia, effective adaptive immune responses are suppressed through the activation of several pathways. For example, the differentiation and activation of dendritic cells, which are the key initiators of adaptive immune responses, are inhibited by signals (such as IL-10) present in the tumour microenvironment. In addition, tumours are frequently infiltrated by regulatory $\mathrm{T}$ cells, which suppress both adaptive and innate immune responses. And MDSCs proliferate in tumour-bearing hosts; these cells, as well as conventional TAMs, are potent suppressors of antitumour immunity ${ }^{46,47}$. Thus, in cancer-related inflammation, multiple pathways are set in motion in to suppress effective antitumour immunity in established tumours. The interplay between these pathways, their hierarchy, and whether they can be targeted for therapy remain largely to be determined.

\section{Cancer-related inflammation and sex hormones}

Sex steroid hormones mediate a classic, clinically relevant pathway of tumour promotion in breast and prostate cancer and have been a target for therapeutic intervention since George Beatson's discovery of hormonedependent breast cancer at the end of the nineteenth century ${ }^{59}$.

Recent studies, however, have uncovered an unexpected relationship between sex steroid hormones and cancer (Fig. 3). For carcinoma of the prostate, which is an androgen-dependent tumour, sensitivity to stimulation with hormones is regulated by selective androgen-receptor modulators. The inflammatory cytokine IL- $1 \beta$, which is produced by macrophages in the tumour microenvironment, converts these receptor modulators from being inhibitory to stimulatory ${ }^{60}$. It has long been known that females are less susceptible to cancer at sites, such as the liver, that are not conventional target organs of sex steroid hormones. After studies of a mouse model of liver carcinogenesis, Willscott Naugler et al. ${ }^{61}$ recently reported that the sex difference in tumour susceptibility resulted from a downregulation of IL- 6 production by macrophages in response to oestrogens. In addition, in male mice, IL- 6 production was triggered to a much greater extent in response to carcinogen-mediated tissue damage (which induces IL-6 production by activating MyD88-dependent TLR- and/or IL-1-receptor signalling pathways). Thus, connections are emerging between the two classic tumour-promoting pathways - inflammation and sex steroid hormones - bringing together the pioneering efforts of Beatson and Rudolf Virchow in studying inflammatory cells in tumours.

\section{Inflammatory pathways in invasion and metastasis}

Most studies of the mechanisms of cancer-related inflammation have focused on the early stages of cancer, but inflammatory mediators and cells are also involved in the migration, invasion and metastasis of malignant cells.

Chemokine receptors and their ligands direct the movement of cells during inflammation, cancer and the maintenance of tissue homeostasis, by affecting cell motility, invasiveness and survival ${ }^{16}$. On transformation, many cells start to express chemokine receptors and thereby use chemokines to aid in their migration to, and survival at, sites that are distant from the original tumour ${ }^{16,62,63}$. For example, the chemokine receptor CXCR4 and its ligand CXCL12 are important for cell movement in both homeostatic and disease states ${ }^{63}$. CXCR4 is frequently expressed by malignant cells ${ }^{16}$, and the amount of CXCR 4 expressed by primary human tumours correlates with the extent to which metastasis to the lymph nodes occurs in colorectal, breast, liver and oesophageal cancer $^{64-66}$. Other functional chemokine receptors (including $\mathrm{CX}_{3} \mathrm{C}$ chemokine receptor $1\left(\mathrm{CX}_{3} \mathrm{CR} 1\right)$, CC-chemokine receptor 1 (CCR1), CCR7, CCR9, CCR10, CXCR1, CXCR2, CXCR3, CXCR5 and CXCR7) are also expressed by malignant cells from a variety of tissues and are implicated in organ-specific metastasis ${ }^{67-72}$; for example, the expression of CCR7 correlates with lymph-node metastasis, and expression of CCR9 with metastasis of melanoma to the small intestine. Malignant melanoma cells express many of the above receptors, perhaps explaining why melanomas are highly metastatic.

How do malignant cells acquire the ability to express chemokine receptors? Several mechanisms have been defined. Autocrine and paracrine extracellular signals, as well as genetic and epigenetic alterations, might each contribute to this change. For example, the previously discussed mutation in VHL and the chromosomal rearrangement that affects RET induce the expression of CXCR4 on initiated cells. Regardless of the mechanism, it is clear that acquisition of chemokine-receptor expression is a common attribute of malignant cells of epithelial or mesenchymal origin that do not normally express these receptors and that this can occur even at the early stages of malignancy ${ }^{65}$.

The invasive capacity of malignant cells can increase in the presence of inflammatory cytokines such as TNF- $\alpha$, IL-1 $\beta$ and IL-6 (ref. 2), possibly as a result of the upregulation of chemokine-receptor expression elicited by these cytokines ${ }^{73}$. For example, autocrine TNF- $\alpha$-mediated signalling upregulates the expression of functional CXCR4 by ovarian cancer cells $^{74}$, and stable knockdown of mRNA encoding this cytokine reduces the expression of both CXCR4 and its ligand CXCL12 by the malignant cells, inhibiting colonization of the peritoneal cavity, angiogenesis and spread to sites distant from the peritoneal cavity ${ }^{73}$. TNF- $\alpha$ is also a potent stimulator of epithelial-mesenchymal transition by breast cancer cells $\mathrm{s}^{75}$, as is activation of NF- $\mathrm{kB}$ signalling.

A further link between NF- $\kappa$ B signalling and metastasis was found by studying a genetic model of prostate cancer in mice, in which inactivation of the gene encoding a major component of the NF- $\kappa \mathrm{B}$ signalling pathway, IKK- $\alpha$, was found to reduce metastatic spread ${ }^{76}$. The mechanism by which this occurs was found to involve activation of receptor activator of NF- $\kappa B$ (RANK) at the surface of malignant prostate epithelial cells in a paracrine manner, by RANK ligand derived from the leukocytes infiltrating the primary tumours. IKK- $\alpha$, which is involved in the RANK signalling pathway, then inhibited expression of the metastasis-suppressor protein maspin, hence promoting the metastatic phenotype. Therefore, removing IKK- $\alpha$ had the opposite effect. It would be interesting to determine whether 
a Normal epithelium

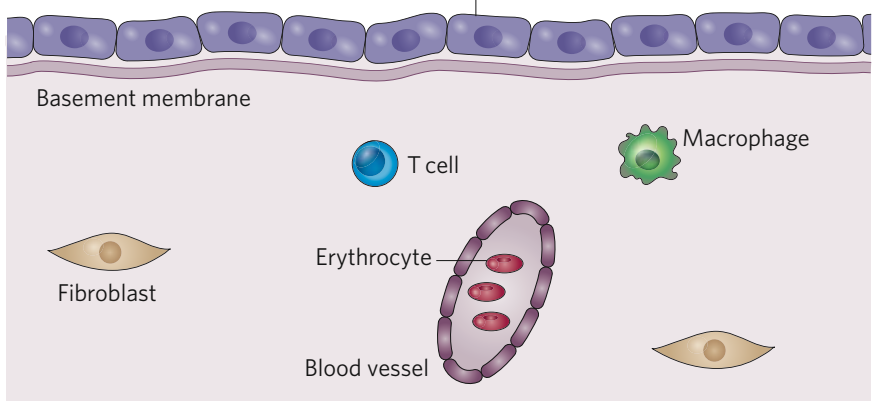

b Carcinoma in situ

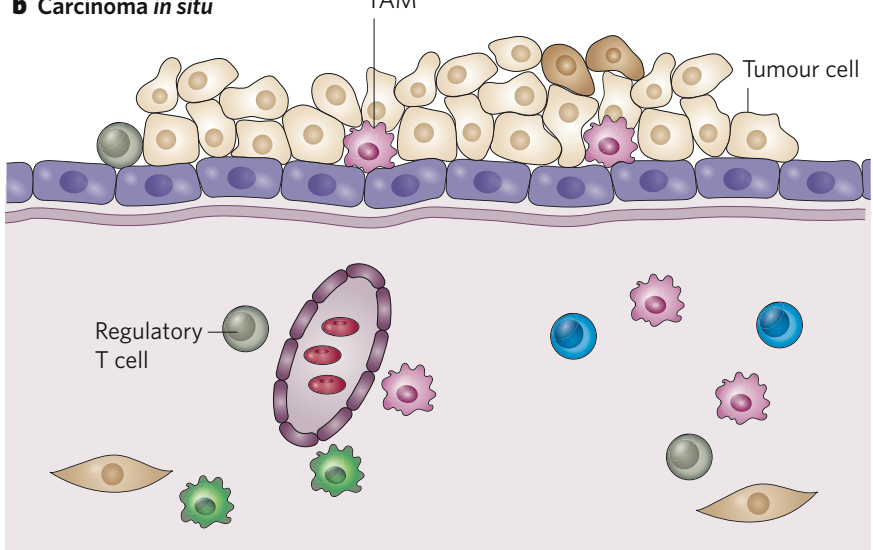

c Local invasion
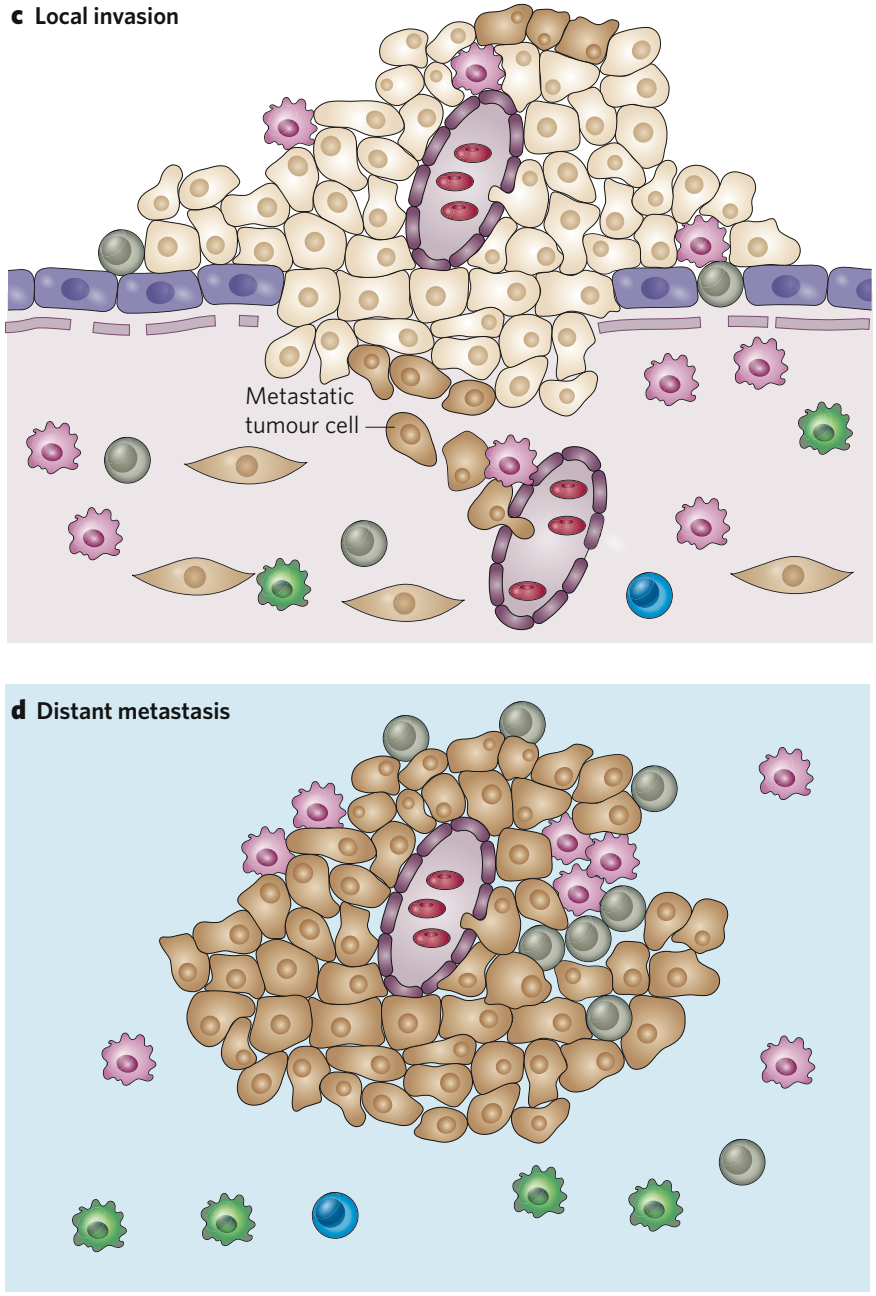

Figure $4 \mid$ Inflammation and the malignant progression of epithelial tumours. a, Normal epithelium. The proliferation of epithelial cells and the homeostatic trafficking of leukocytes in the epithelium is regulated by autocrine and paracrine chemokine- and cytokine-mediated signalling. b, Carcinoma in situ. The extrinsic (inflammatory) pathway and the intrinsic (oncogenic) pathway induce the production of chemokines and cytokines. These factors attract a tumour-promoting infiltrate (which contains, for example, TAMs and regulatory T cells, as shown here), and they also promote angiogenesis. The expression of chemokine receptors can be induced on initiated cells (that is, early tumour cells). These receptors then aid in tumourcell survival and might be necessary (but are not sufficient) for invasion across the basement membrane. Both autocrine and paracrine networks of cytokines and chemokines are involved in these processes. c, Local invasion. The chemokines and cytokines continue to attract and modulate a tumourpromoting infiltrate. They also promote angiogenesis and control tissue remodelling (for example, changes in the basement membrane). Operating in paracrine and autocrine loops, these factors induce the expression of genes associated with survival, invasion and migration in cells that have enough oncogenic changes to allow them to invade the basement membrane. The chemokines and cytokines are also involved in the intravasation of tumour cells into blood vessels and in lymphatic spread. d, Distant metastasis. The autocrine and paracrine chemokine- and cytokine-mediated signalling promotes the survival of malignant cells in distant organs, again attracting a tumour-promoting infiltrate and stimulating angiogenesis.

chemokine ligands and their receptors also contribute to the effects of IKK- $\alpha$ and whether IKK- $\alpha$ is implicated in other metastatic pathways.

Other cells within the tumour also affect processes in the later stages of cancer. TAMs have been described as "obligate partners for tumour-cell migration, invasion and metastasis" "77. The first experiments to show this conclusively involved a genetic model of breast cancer in macrophage-deficient mice ${ }^{44}$. The tumours developed normally but were unable to form pulmonary metastases in the absence of macrophages. The mechanism by which metastasis occurs in this case involves a paracrine loop of tumour-cell M-CSF and macrophage epidermal growth factor ${ }^{44}$, with intravasation assisted by direct interactions between tumour cells and $\mathrm{TAMs}^{78}$.

In addition, inflammatory macrophages increase peritoneal dissemination of tumour cells and metastatic spread in an ovarian cancer model ${ }^{79}$. The ability of macrophages to aid in ovarian tumour-cell migration and invasion can also be modelled in vitro ${ }^{48,80}$. Co-culture of macrophages with tumour cells was shown to increase their invasive capacity in an NF- $\kappa B$-dependent and TNF- $\alpha$-dependent manner.

In summary, chemokines and cytokines coordinate autocrine and paracrine interactions between malignant cells and infiltrating leukocytes. These interactions increase the migration, invasion and survival of malignant cells. They also affect the growth of the primary tumour and the ability of tumour cells to colonize the metastatic niche ${ }^{16,63,67,81}$ (Fig. 4).

\section{Cancer-inhibitory inflammation}

Although numerous experimental and clinical results point to inflammation having protumour activity, some evidence does not fit into this general pattern. For example, a marked chronic inflammatory response such as that in psoriasis is not associated with an increased risk of developing skin cancer ${ }^{82}$. Also, in certain tumours or subsets of tumours, the presence of inflammatory cells is associated with better prognosis (for example, eosinophils in colon tumours, and TAMs in a subset of breast tumours and pancreatic tumours). These observations are likely to reflect that inflammatory cells can destroy tumour cells, in addition to normal tissue cells ${ }^{41}$. For example, appropriately activated macrophages, a prototypical component of cancer-related inflammation, can kill tumour cells and elicit cancer-destructive inflammatory responses centred on the blood-vessel wall, although in most cases their tumour-promoting properties prevail ${ }^{41}$. Evidence indicates that $\mathrm{NF}-\kappa \mathrm{B}$ is important in determining this balance between the protumour and antitumour properties of macrophages ${ }^{37,83}$, thus NF- $\kappa B$ could be targeted to 're-educate' tumour-promoting macrophages towards an antitumour function. 
Box 2 | Unanswered questions about cancer-related inflammation

1. Is inflammation sufficient for cancer development?

2. Despite the diversity of tumours and oncogenic pathways, are there aspects of cancer-related inflammation that are common to all malignancies?

3. How can the balance between 'bad' inflammation and 'good' inflammation be altered to favour adaptive immunity instead of tumour development?

4. What is the relationship between MDSCs and TAMs?

5. What is the clinical relevance of the connections between sex steroid hormones and inflammation?

6. What is the best way to target cancer-related inflammation in patients with cancer? This is the most difficult question.

The importance of this balance is evident in psoriasis. Psoriasis is a T helper $1\left(\mathrm{~T}_{\mathrm{H}} 1\right)$-cell-mediated disease that involves a massive accumulation of neutrophils and monocytes in the skin, the latter of which are likely to become macrophages with an M1 phenotype, which have antitumour activity. Hence, the type of inflammation found in psoriasis does not promote the development of skin cancers because of the presence of macrophages that can destroy any nascent tumour cells. The dual potential of cancer-related inflammation (cancer promoting versus cancer inhibiting) may also be affected by the tissue type. In a skin tumour model, the overexpression of NF- $\mathrm{KB}$ was found to inhibit invasive epidermal neoplasia ${ }^{84}$, whereas blocking NF- $\kappa B$ activity inhibited the development of experimental liver and colon cancers ${ }^{31,32}$.

The concept that the activation of innate immune responses can promote a protective response to cancer is not new. In the late nineteenth century, William Coley noted that some patients with cancer who had severe postoperative infections at the tumour site underwent spontaneous and sustained tumour regression ${ }^{85}$. He then developed Coley's mixed toxins, a filtrate from cultures of Streptococcus pyogenes and Serratia marcescens, which was administered into the tumour or the surrounding tissues in patients with a range of advanced cancers. Although both the technique and the results were controversial, even at the time, Coley documented cases of the long-term survival of individuals with malignancies that remain a major challenge to treat now. And even before Coley's time, there was evidence for the regression of cancer after certain bacterial infections. More recently, this approach was adapted successfully to treat patients with bladder cancer by administering Mycobacterium bovis bacillus Calmette-Guérin (BCG). Such treatments probably trigger a 'good' inflammatory response (through TLRs) that not only promotes the differentiation of monocytes into macrophages with an M1 phenotype but also promotes the development of a sustained and effective adaptive immune response to the tumour. This type of response might also contribute to successful chemotherapy or radiotherapy, according to recent data obtained by Lionel Apetoh and colleagues ${ }^{86}$. After treating experimentally induced breast cancers, the authors found that dying tumour cells were able to cross-present antigen to dendritic cells in a TLR4- and MyD88-dependent manner, as well as trigger protective immune responses through a 'danger signal' (HMGB1), again by signalling through TLR4. However, when tumours were grown in mice with a mutant TLR4, the efficacy of chemotherapy and radiotherapy was reduced. Moreover, patients with breast cancer who have a mutation in TLR4 were found to have a higher frequency of metastasis.

The exact mechanisms by which a 'good' immune response can be reliably triggered during anticancer therapy are not entirely clear. It will be important to find the optimal stimuli to change a tumour-promoting ( $\mathrm{T}_{\mathrm{H}} 2$ cell and $\mathrm{M} 2$ macrophage) microenvironment to a tumourinhibiting $\left(\mathrm{T}_{\mathrm{H}} 1\right.$ cell and $\mathrm{M} 1$ macrophage) microenvironment, and to understand the signalling mechanisms involved.

\section{The big questions}

The connection between inflammation and cancer is now generally accepted, but several questions remain. Some of these outstanding questions are listed in Box 2 and discussed in detail in this section.
First, it is unclear whether inflammation is sufficient for the development of cancer. That is, can inflammation cause neoplasia in the absence of an exogenous carcinogenic agent? Several lines of evidence provide hints that it can. In a mouse model of bowel inflammation caused by IL-10 deficiency, the frequency of DNA mutations observed in the colon in the absence of exogenous carcinogens was 4-5-fold greater than in IL-10sufficient mice ${ }^{87}$. In addition, a comparison of human tumours and the appropriate normal tissues showed a higher frequency of random mutations in tumour cells ${ }^{88}$. The only exception to the exceedingly low frequency of random mutations found in normal cells $\left(<1 \times 10^{-8}\right.$ per base pair) was an inflamed tissue ${ }^{88}$. One candidate for an endogenous inflammatory carcinogen' is reactive oxygen species. Neutrophils, for example, have been shown to inhibit base-excision repair in an alveolar epithelial cell line, an effect that is mediated by a product of myeloperoxidase activity, hypochlorous acid ${ }^{89}$. Interestingly, a polymorphism in the promoter of the gene encoding myeloperoxidase has been associated with resistance to the development of lung cancer in smokers ${ }^{90}$. Therefore, although the evidence ${ }^{87-91}$ suggests that inflammation causes cancer, formal proof is still required.

The second issue is that diversity and plasticity are characteristics of chronic inflammation and its main orchestrator, macrophages. Studies using classic histological techniques and a variety of model systems show that cancer-related inflammation differs between tumour types. It will be important to define which cellular and molecular components are common to all cancer-promoting inflammatory responses, and which are specific to particular tissues and tumour types.

The third issue relates to tipping the balance between cancerpromoting inflammatory responses and cancer-inhibiting inflammatory responses. A key point that needs to be addressed is how to activate an appropriate antitumour adaptive immune response.

The fourth open question relates to MDSCs, which have recently emerged as an important factor in cancer-related inflammation ${ }^{46}$. The definition of MDSCs is operational and includes a heterogeneous set of cells in the peripheral blood and spleen. Are these cells a distinct population, or do they belong to a continuum of TAM differentiation?

Fifth, the emergence of a connection between sex steroid hormones, inflammation and cancer is a major conceptual advance that provides the first link between these two classic tumour-promoting pathways ${ }^{92}$. This connection between inflammation and sex steroid hormones probably reflects the interplay between inflammation and hormones during reproduction. Analysing this link in human cancer and its implications for resistance to hormonal therapies could have a huge impact in the clinic, given the widespread use of selective androgen-receptor modulators and selective oestrogen-receptor modulators (for example, tamoxifen) to inhibit the growth-stimulating effects of sex hormones on prostate cancer and breast cancer, respectively.

Last, the biggest question is whether knowledge about cancer-related inflammation can be translated into useful approaches to preventing, diagnosing and treating cancer. Malignant cells are 'moving targets' that can become resistant to even the most sophisticated targeted drugs. Using a combination therapy that attacks both malignant cells and the 'other half' of the tumour mass (that is, the inflammatory cells) could be more effective and might elicit long-lasting adaptive immunity to the transformed cells.

Many drugs that could target cancer-related inflammation - for example, chemokine-receptor antagonists and cytokine-receptor antagonists, and COX inhibitors - are in clinical trials for other diseases. In terms of cancer, phase I/II trials of antagonists of IL-6, the IL-6 receptor, CCL2, CCR4 and CXCR4 are underway for a range of epithelial and haematopoietic malignancies. The first (phase I/II) clinical trials of TNF- $\alpha$ antagonists in patients with advanced cancer have resulted in disease stabilization and some partial responses ${ }^{93-95}$, particularly for those with renal-cell carcinoma ${ }^{95}$. Also, a structural analogue of thalidomide, lenalidomide, that inhibits production of several inflammatory cytokines has been shown to be active against advanced myeloma when combined with dexamethasone ${ }^{96}$. In addition, COX2 inhibitors have been shown to prevent the recurrence of both sporadic adenomatous 
polyps and adenomas in people with a genetic predisposition to developing these $e^{97,98}$.

Drugs that target cancer-related inflammation have the potential to re-educate a tumour-promoting inflammatory infiltrate or to prevent such cells from migrating to the tumour site. They also might be able to 're-align' a tumour-promoting microenvironment to become a tumourinhibiting microenvironment, to encourage tumour-specific adaptive immune responses and to inhibit metastatic spread. This potential for reversing tumour-supporting inflammation could be the start of an exciting new era for anticancer therapies.

1. Balkwill, F. \& Mantovani, A. Inflammation and cancer: back to Virchow? Lancet 357, 539-545 (2001).

This paper revisits Virchow's legacy and highlights the connections between inflammation and cancer.

2. Balkwill, F., Charles, K. A. \& Mantovani, A. Smoldering and polarized inflammation in the initiation and promotion of malignant disease. Cancer Cell 7, 211-217 (2005).

3. Coussens, L. M. \& Werb, Z. Inflammation and cancer. Nature $420,860-867$ (2002).

4. Karin, M. Nuclear factor- $\mathrm{kB}$ in cancer development and progression. Nature $441,431-436$ (2006).

5. Koehne, C. H. \& Dubois, R. N. COX-2 inhibition and colorectal cancer. Semin. Oncol. 31, 12-21(2004).

6. Flossmann, E. \& Rothwell, P. M. Effect of aspirin on long-term risk of colorectal cancer: consistent evidence from randomised and observational studies. Lancet 369, 1603-1613 (2007).

7. Chan, A. T., Ogino, S. \& Fuchs, C. S. Aspirin and the risk of colorectal cancer in relation to the expression of COX-2. N. Engl. J. Med. 356, 2131-2142 (2007).

8. Borrello, M. G. et al. Induction of a proinflammatory program in normal human thyrocytes by the RET/PTC1 oncogene. Proc. Natl Acad. Sci. USA 102, 14825-14830 (2005). This is the first report that a frequent genetic event that causes cancer in humans (rearrangement of the chromosome on which RET is located, in human papillary thyroid carcinoma) activates an inflammatory transcriptional program in normal cells that is associated with metastatic behaviour.

9. De Falco, V. et al. Biological role and potential therapeutic targeting of the chemokine receptor CXCR4 in undifferentiated thyroid cancer. Cancer Res. 67, 11821-11829 (2007).

10. Xu, K. \& Shu, H. K. EGFR activation results in enhanced cyclooxygenase-2 expression through p38 mitogen-activated protein kinase-dependent activation of the Sp1/Sp3 transcription factors in human gliomas. Cancer Res. 67, 6121-6129 (2007).

11. Guerra, C. et al. Chronic pancreatitis is essential for induction of pancreatic ductal adenocarcinoma by K-Ras oncogenes in adult mice. Cancer Cell 11, 291-302 (2007).

12. Sparmann, A. \& Bar-Sagi, D. Ras-induced interleukin-8 expression plays a critical role in tumor growth and angiogenesis. Cancer Cell 6, 447-458 (2004).

13. Sumimoto, H., Imabayashi, F., Iwata, T. \& Kawakami, Y. The BRAF-MAPK signaling pathway is essential for cancer-immune evasion in human melanoma cells. J. Exp. Med. 203, 1651-1656 (2006)

14. Shchors, K. et al. The Myc-dependent angiogenic switch in tumors is mediated by interleukin $1 \beta$. Genes Dev. 20, 2527-2538 (2006).

15. Soucek, L. et al. Mast cells are required for angiogenesis and macroscopic expansion of Myc-induced pancreatic islet tumors. Nature Med. 13, 1211-1218 (2007)

16. Balkwill, F. Cancer and the chemokine network. Nature Rev. Cancer 4, 540-550 (2004).

17. Kobielak, A. \& Fuchs, E. Links between a-catenin, NF- $\kappa B$, and squamous cell carcinoma in skin. Proc. Natl Acad. Sci. USA 103, 2322-2327 (2006).

18. Phillips, R. J. et al. Epidermal growth factor and hypoxia-induced expression of CXC chemokine receptor 4 on non-small cell lung cancer cells is regulated by the phosphatidylinositol 3-kinase/PTEN/AKT/mammalian target of rapamycin signaling pathway and activation of hypoxia inducible factor-1a. J. Biol. Chem. 280, 22473-22481 (2005).

19. Schioppa, T. et al. Regulation of the chemokine receptor CXCR4 by hypoxia. J. Exp. Med. 198, 1391-1402 (2003).

20. Staller, P. et al. Chemokine receptor CXCR4 downregulated by von Hippel-Lindau tumour suppressor pVHL. Nature 425, 307-311 (2003).

21. Bierie, B. \& Moses, H. L. TGF- $\beta$ and cancer. Cytokine Growth Factor Rev. 17, 29-40 (2006).

22. Yu, H., Kortylewski, M. \& Pardoll, D. Crosstalk between cancer and immune cells: role of STAT3 in the tumour microenvironment. Nature Rev. Immunol. 7, 41-51 (2007).

23. Voronov, E. et al. IL-1 is required for tumor invasiveness and angiogenesis. Proc. Natl Acad. Sci. USA 100, 2645-2650 (2003)

24. Grivennikov, S. \& Karin, M. Autocrine IL-6 signaling: a key event in tumorigenesis? Cancer Cell 13, 7-9 (2008)

25. Szlosarek, P. W. \& Balkwill, F. R. Tumour necrosis factor $\alpha$ : a potential target for the therapy of solid tumours. Lancet Oncol. 4, 565-573 (2003)

26. Langowski, J. L. et al. IL-23 promotes tumour incidence and growth. Nature $442,461-465$ (2006).

27. Courtois, G. \& Gilmore, T. D. Mutations in the NF- $\kappa B$ signaling pathway: implications for human disease. Oncogene 25, 6831-6843 (2006).

28. Carbia-Nagashima, A. et al. RSUME, a small RWD-containing protein, enhances SUMO conjugation and stabilizes HIF-1a during hypoxia. Cell 131, 309-323 (2007).

29. Mizukami, Y. et al. Induction of interleukin-8 preserves the angiogenic response in $\mathrm{HIF-1 \alpha -}$ deficient colon cancer cells. Nature Med. 11, 992-997 (2005).

30. Rius, J. et al. NF-kB links innate immunity to the hypoxic response through transcriptional regulation of HIF-1a. Nature 453, 807-811 (2008).

31. Greten, F. R. et al. IKK $\beta$ links inflammation and tumorigenesis in a mouse model of colitisassociated cancer. Cell 118, 285-296 (2004).

32. Pikarsky, E. et al. NF- $\mathrm{kB}$ functions as a tumour promoter in inflammation-associated cancer. Nature 431, 461-466 (2004).
References 31 and 32 provide evidence that NF- $\mathrm{kB}$ is an endogenous promoter of colon and liver carcinogenesis. Reference 31 also shows that NF- $\mathrm{kB}$ activation in myeloid cells is required for colitis-associated cancer.

33. Maeda, S., Kamata, H., Luo, J. L., Leffert, H. \& Karin, M. IKK $\beta$ couples hepatocyte death to cytokine-driven compensatory proliferation that promotes chemical hepatocarcinogenesis. Cell 121, 977-990 (2005)

34. Garlanda, C. et al. Increased susceptibility to colitis-associated cancer of mice lacking TIR8, an inhibitory member of the interleukin-1 receptor family. Cancer Res. 67, 6017-6021 (2007).

35. Xiao, H. et al. The Toll-interleukin-1 receptor member SIGIRR regulates colonic epithelial homeostasis, inflammation, and tumorigenesis. Immunity 26, 461-475 (2007).

36. Biswas, S. K. et al. A distinct and unique transcriptional program expressed by tumorassociated macrophages: defective NF- $\mathrm{kB}$ and enhanced IRF-3/STAT1 activation. Blood 107, 2112-2122 (2006).

37. Saccani, A. et al. p50 nuclear factor-kB overexpression in tumor-associated macrophages inhibits M1 inflammatory responses and antitumor resistance. Cancer Res. 66, 1143211440 (2006)

38. Bromberg, J. F. et al. Stat3 as an oncogene. Cell 98, 295-303 (1999).

39. Wang, T. et al. Regulation of the innate and adaptive immune responses by Stat-3 signaling in tumor cells. Nature Med. 10, 48-54 (2004).

40. Kortylewski, M. et al. Inhibiting Stat3 signaling in the hematopoietic system elicits multicomponent antitumor immunity. Nature Med. 11,1314-1321 (2005)

41. Mantovani, A., Bottazzi, B., Colotta, F., Sozzani, S. \& Ruco, L. The origin and function of tumor-associated macrophages. Immunol. Today 13, 265-270 (1992).

42. Coussens, L. M., Tinkle, C. L., Hanahan, D. \& Werb, Z. MMP-9 supplied by bone marrowderived cells contributes to skin carcinogenesis. Cell 103, 481-490 (2000).

43. Bunt, S. K. et al. Reduced inflammation in the tumor microenvironment delays the accumulation of myeloid-derived suppressor cells and limits tumor progression. Cancer Res. 67, 10019-10026 (2007).

44. Lin, E. Y., Nguyen, A. V., Russell, R. G. \& Pollard, J. W. Colony-stimulating factor 1 promotes progression of mammary tumors to malignancy. J. Exp. Med. 193, 727-740 (2001). This paper describes the first genetic evidence that TAMs promote cancer, in a study of a primary breast carcinoma model.

45. De Palma, M. et al. Tie2 identifies a hematopoietic lineage of proangiogenic monocytes required for tumor vessel formation and a mesenchymal population of pericyte progenitors. Cancer Cell 8, 211-226 (2005)

46. Sica, A. \& Bronte, V. Altered macrophage differentiation and immune dysfunction in tumor development. J. Clin. Invest. 117, 1155-1166 (2007)

47. Mantovani, A., Sozzani, S., Locati, M., Allavena, P. \& Sica, A. Macrophage polarization: tumor-associated macrophages as a paradigm for polarized M2 mononuclear phagocytes. Trends Immunol. 23, 549-555 (2002)

48. Hagemann, T. et al. Ovarian cancer cells polarize macrophages toward a tumor-associated phenotype. J. Immunol. 176, 5023-5032 (2006)

49. Fischer, C. et al. Anti-PIGF inhibits growth of VEGFR-inhibitor-resistant tumors without affecting healthy vessels. Cell 131, 463-475 (2007)

50. Kaplan, R. N. et al. VEGFR1-positive haematopoietic bone marrow progenitors initiate the pre-metastatic niche. Nature 438, 820-827 (2005)

51. Shojaei, F. et al. Bv8 regulates myeloid-cell-dependent tumour angiogenesis. Nature 450 , 825-831 (2007)

52. Coussens, L. M. et al. Inflammatory mast cells up-regulate angiogenesis during squamous epithelial carcinogenesis. Genes Dev. 13, 1382-1397 (1999).

53. Lewis, C. E., De Palma, M. \& Naldini, L. Tie2-expressing monocytes and tumor angiogenesis: regulation by hypoxia and angiopoietin 2. Cancer Res. 67, 8429-8432 (2007).

54. Sozzani, S., Rusnati, M., Riboldi, E., Mitola, S. \& Presta, M. Dendritic cell-endothelial cell cross-talk in angiogenesis. Trends Immunol. 28, 385-392 (2007).

55. Noonan, D. M., De Lerma Barbaro, A., Vannini, N., Mortara, L. \& Albini, A. Inflammation, inflammatory cells and angiogenesis: decisions and indecisions. Cancer Metastasis Rev. 27, 31-40 (2008).

56. Dunn, G. P., Old, L. J. \& Schreiber, R. D. The immunobiology of cancer immunosurveillance and immunoediting. Immunity 21, 137-148 (2004)

57. Swann, J. B. et al. Demonstration of inflammation-induced cancer and cancer immunoediting during primary tumorigenesis. Proc. Natl Acad. Sci. USA 105, 652-656 (2008).

58. de Visser, K. E., Korets, L. V. \& Coussens, L. M. De novo carcinogenesis promoted by chronic inflammation is B lymphocyte dependent. Cancer Cell 7, 411-423 (2005) This paper shows that in a model of human-papilloma-virus-driven carcinogenesis, adaptive immune responses mediated by B cells coordinate cancer-promoting inflammation.

59. Beatson, G. On the treatment of inoperable cases of carcinoma of the mamma: suggestions for a new method of treatment with illustrative cases. Lancet 2, 104-162, (1896).

60. Zhu, P. et al. Macrophage/cancer cell interactions mediate hormone resistance by a nuclear receptor derepression pathway. Cell 124, 615-629 (2006).

61. Naugler, W. E. et al. Gender disparity in liver cancer due to sex differences in MyD88dependent IL-6 production. Science 317, 121-124 (2007). References 60 and 61 show that two classic pathways of cancer promotion, hormones and inflammation, are linked in both liver cancer and prostate cancer.

62. Muller, A. et al. Involvement of chemokine receptors in breast cancer metastasis. Nature 410, 50-56 (2001)

63. Burger, J. A. \& Kipps, T. J. CXCR4: a key receptor in the crosstalk between tumor cells and their microenvironment. Blood 107, 1761-1767 (2006)

64. Kaifi, J. T. et al. Tumor-cell homing to lymph nodes and bone marrow and CXCR4 expression in esophageal cancer. J. Natl Cancer Inst. 97, 1840-1847 (2005).

65. Salvucci, O. et al. The role of CXCR4 receptor expression in breast cancer: a large tissue microarray study. Breast Cancer Res. Treat. 97, 275-283 (2006).

66. Kim, J. et al. Chemokine receptor CXCR4 expression in colorectal cancer patients increases the risk for recurrence and for poor survival. J. Clin. Oncol. 23, 2744-2753 (2005). 
67. Shields, J. D. et al. Autologous chemotaxis as a mechanism of tumor cell homing to lymphatics via interstitial flow and autocrine CCR7 signaling. Cancer Cell 11, 526-538 (2007).

68. Kawada, K. et al. Pivotal role of CXCR3 in melanoma cell metastasis to lymph nodes. Cancer Res. 64, 4010-4017 (2004).

69. Shulby, S. A., Dolloff, N. G., Stearns, M. E., Meucci, O. \& Fatatis, A. CX ${ }_{3}$ CR1-fractalkine expression regulates cellular mechanisms involved in adhesion, migration, and survival of human prostate cancer cells. Cancer Res. 64, 4693-4698 (2004)

70. Burns, J. M. et al. A novel chemokine receptor for SDF-1 and I-TAC involved in cell survival, cell adhesion, and tumor development. J. Exp. Med. 203, 2201-2213 (2006).

71. Zipin-Roitman, A. et al. CXCL10 promotes invasion-related properties in human colorectal carcinoma cells. Cancer Res. 67, 3396-3405 (2007).

72. Ghadjar, P. et al. Chemokine receptor CCR6 expression level and liver metastases in colorectal cancer. J. Clin. Oncol. 24, 1910-1916 (2006).

73. Kulbe, H. et al. The inflammatory cytokine tumor necrosis factor- $a$ generates an autocrine tumor-promoting network in epithelial ovarian cancer cells. Cancer Res. 67, 585-592 (2007).

74. Kulbe, H., Hagemann, T., Szlosarek, P. W., Balkwill, F. R. \& Wilson, J. L. The inflammatory cytokine tumor necrosis factor-a regulates chemokine receptor expression on ovarian cancer cells. Cancer Res. 65, 10355-10362 (2005).

75. Bates, R. C. \& Mercurio, A. M. Tumor necrosis factor-a stimulates the epithelial-tomesenchymal transition of human colonic organoids. Mol. Biol. Cell 14, 1790-1800 (2003).

76. Luo, J. L. et al. Nuclear cytokine-activated IKKa controls prostate cancer metastasis by repressing maspin. Nature 446, 690-694 (2007).

77. Condeelis, J. \& Pollard, J. W. Macrophages: obligate partners for tumor cell migration, invasion, and metastasis. Cell 124, 263-266 (2006)

78. Wyckoff, J. B. et al. Direct visualization of macrophage-assisted tumor cell intravasation in mammary tumors. Cancer Res. 67, 2649-2656 (2007).

79. Robinson-Smith, T. M. et al. Macrophages mediate inflammation-enhanced metastasis of ovarian tumors in mice. Cancer Res. 67, 5708-5716 (2007).

80. Hagemann, T. et al. Macrophages induce invasiveness of epithelial cancer cells via NF-кB and JNK. J. Immunol. 175, 1197-1205 (2005).

81. Marchesi, F. et al. Increased survival, proliferation, and migration in metastatic human pancreatic tumor cells expressing functional CXCR4. Cancer Res. 64, 8420-8427 (2004).

82. Nickoloff, B. J., Ben-Neriah, Y. \& Pikarsky, E. Inflammation and cancer: is the link as simple as we think? J. Invest. Dermatol. 124, x-xiv (2005).

83. Hagemann, T. et al. Re-educating tumor-associated macrophages by targeting NF-кB. J. Exp. Med. 205, 1261-1268 (2008)

This paper shows that NF-KB activated through the IL-1 receptor and MyD88 signalling pathway maintains the phenotype of TAMs, suggesting that tumour-promoting macrophages might be re-educated by the targeting of NF- $\kappa \mathrm{B}$.

84. Dajee, M. et al. NF-kB blockade and oncogenic Ras trigger invasive human epidermal neoplasia. Nature 421, 639-643 (2003)
85. Coley, W. B. The treatment of malignant tumors by repeated inoculations of erysipelas: with a report of ten original cases. Am. J. Med. Sci. 105, 487-511 (1893).

86. Apetoh, L. et al. Toll-like receptor 4-dependent contribution of the immune system to anticancer chemotherapy and radiotherapy. Nature Med. 13, 1050-1059 (2007).

87. Sato, Y. et al. IL-10 deficiency leads to somatic mutations in a model of IBD. Carcinogenesis 27, 1068-1073 (2006)

88. Bielas, J. H., Loeb, K. R., Rubin, B. P., True, L. D. \& Loeb, L. A. Human cancers express a mutator phenotype. Proc. Natl Acad. Sci. USA 103, 18238-18242 (2006)

89. Gungor, N., Godschalk, R. W. L. Pachen, D. M. Van Schooten, F.J. \& Knaapen, A. M. Activated neutrophils inhibit nucleotide excision repair in human pulmonary epithelial cells: role of myeloperoxidase. FASEB J. 21, 2359-2367 (2007).

90. Dally, $\mathrm{H}$. et al. Myeloperoxidase (MPO) genotype and lung cancer histologic types: the MPO -463 A allele is associated with reduced risk for small cell lung cancer in smokers. Int. J. Cancer 102, 530-535 (2002).

91. Rao, V. P. et al. Innate immune inflammatory response against enteric bacteria Helicobacter hepaticus induces mammary adenocarcinoma in mice. Cancer Res. 66, 7395-7400 (2006)

92. Mantovani, A. Cancer: an infernal triangle. Nature 448, 547-548 (2007).

93. Madhusudan, S. et al. Study of etanercept, a tumor necrosis factor- $a$ inhibitor, in recurrent ovarian cancer. J. Clin. Oncol. 23, 5950-5959 (2005)

94. Brown, E. R. et al. A clinical study assessing the tolerability and biological effects of infliximab, a TNF- $\alpha$ inhibitor, in patients with advanced cancer. Ann. Oncol. 19, 1340-1346 (2008).

95. Harrison, M. L. et al. Tumor necrosis factor a as a new target for renal cell carcinoma: two sequential phase II trials of infliximab at standard and high dose. J. Clin. Oncol. 25 4542-4549 (2007)

The paper reports the first clinical evidence that TNF- $\alpha$ could be a target for treating renal-cell carcinoma.

96. Weber, D. M. et al. Lenalidomide plus dexamethasone for relapsed multiple myeloma in North America. N. Engl. J. Med. 357, 2133-2142 (2007).

97. Bertagnolli, M. M. et al. Celecoxib for the prevention of sporadic colorectal adenomas. N. Engl. J. Med. 355, 873-884 (2006).

98. Steinbach, G. et al. The effect of celecoxib, a cyclooxygenase-2 inhibitor, in familial adenomatous polyposis. N. Engl. J. Med. 342, 1946-1952 (2000).

Acknowledgements A.M., P.A. and A.S. are supported by the Italian Association for Cancer Research, the Italian Ministry of Health, the Italian Ministry of Universities and Research, and the European Commission. F.B. is supported by Cancer Research UK, the Medical Research Council, the Association for International Cancer Research and the Higher Education Funding Council for England.

Author Information Reprints and permissions information is available at wWw.nature.com/reprints. The authors declare no competing financial interests. Correspondence should be addressed to A.M. (alberto.mantovani@humanitas.it). 